\title{
Pentacene devices: molecular structure, charge transport and photo response
}

\author{
Bert Nickel $^{* 1}$, Matthias Fiebig ${ }^{1}$, Stefan Schiefer ${ }^{1}$, Martin Göllner ${ }^{1}$, Martin Huth ${ }^{1}$, \\ Christoph Erlen ${ }^{2}$, and Paolo Lugli ${ }^{2}$ \\ ${ }^{1}$ Ludwig-Maximilians-Universität, Department für Physik and CeNS, Geschwister-Scholl-Platz 1, D- \\ 80539 München \\ 2 Technische Universität München, Institute for Nanoelectronics, Arcisstrasse 21, D-80333 München
}

Received 20/11/2007

Key words pentacene, OTFT, structure, synchrotron, defect density PACS 04A25

In this feature article, we will discuss to which extend the peculiar growth properties of pentacene on metallic contacts and on gate dielectrics contribute to the device performance of organic thin film transistors. The early growth state of pentacene monolayers is reviewed, as well as the molecular structure of the so called thin film phase. Then, the relation of structural defect densities to trap densities is discussed. The spatially resolved photo response of a pentacene transistor will be presented in the context of injection barriers and contact homogeneity. 


\section{Introduction}

Today, the field of organic electronics is wide open [1] [2]. While organic light emitting diodes (OLEDs) have already successfully entered the market, the roadmap for integrated circuits is still not settled. Mobilities $\mu$ for pentacene organic thin film transistors (OTFTs) commonly ranging between 0.1 and 1 $\mathrm{cm} / \mathrm{Vs}$ have been reproduced by many groups world wide $[3,4,5,6]$. In spite of the enormous activities in synthesizing and screening for new materials for transistor applications, pentacene has successfully defended its leading position for the production of OTFTs.

The reason for pentacene being superior for the production of TFT devices [7, 8] when compared to other molecules [9] is still not obvious. In this feature article, we will discuss to which extend the peculiar growth properties [10] of pentacene on metallic contacts and gate dielectrics contribute to the device performance. For this purpose, first the early growth state of pentacene films and the molecular structure of the so called thin film phase is reviewed. Then, major sources of crystal defects in thin films as determined by advanced synchrotron diffraction techniques are discussed. The relation of these defects to the frequently discussed electronic traps that strongly influence transport properties of TFTs [6, $11,12]$ is indicated. Finally, the spatially resolved photo response of pentacene OTFTs will be discussed in the context of injection barriers and contact homogeneity.

\section{Pentacene thin films}

\subsection{Film formation on inert surfaces}

Pentacene can be evaporated quite conveniently by resistive heating of a tantalum crucible filled with purified pentacene powder. This procedure is ultra high vacuum compatible and a molecular beam with typical deposition rates ranging from below $0.01 \mathrm{~nm} / \mathrm{s}$ up to $1 \mathrm{~nm} / \mathrm{sec}$ can be easily achieved [13], as verified by a quartz micro balance at the sample position. Also alternative methods such as laser evaporation have been reported [14]. Growth temperatures typically range from room temperature up to $50^{\circ} \mathrm{C}$. At higher temperatures, a much higher deposition rate is needed to compensate for desorption. Thus, with MBE techniques, the maximum growth temperature is limited to about $75^{\circ} \mathrm{C}$. A typical example for a pentacene film grown on a thermal oxide with Au bottom contacts is shown in Fig. 1a. If substrate and growth conditions are properly chosen, a continuous film forms on $\mathrm{SiO}_{2}$ [Fig. 1a] with grains of the order of several microns which exhibit step heights comparable to the length of the pentacene molecule (Fig. 1b). On the gold contacts, the film morphology is much more rugged (Fig. 1a). The reason for the profound difference in growth behavior on $\mathrm{SiO}_{2}$ and $\mathrm{Au}$ is discussed below.
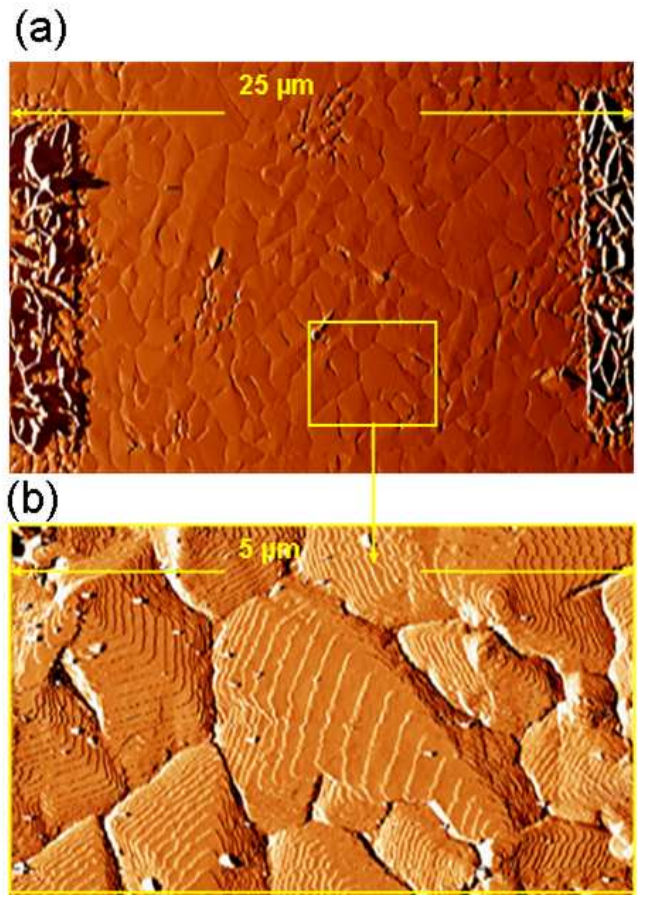

Fig. 1 AFM amplitude micrograph of a pentacene OTFT structure. (a) Gold contacts on $\mathrm{SiO}_{2}$ have been covered by a $50 \mathrm{~nm}$ pentacene film. (b) A zoom reveals a grainy structure and terraces with step heights of ca. $15.4 \AA$

The initial stage of pentacene thin film growth on $\mathrm{SiO}_{2}$ can be described by diffusion limited aggregation (DLA) [15]. In this growth mode, incoming molecules initially diffuse across the surface. Once they meet a critical number of molecules, they form a stable nuclei, which subsequently grows in area during deposition. The submonolayer islands stemming from the growth of such nuclei exhibit a fractal shape, which is the fingerprint of this growth mode. Pentacene submonolayers grown on bare $\mathrm{Si}$ 
surfaces show such fractal shapes, as revealed first by in-situ photo electron emission microscopy experiments [16]. Subsequent AFM studies have shown that the diffusion length on silicon oxide is significantly reduced [17] compared to e.g. H-terminated $\mathrm{Si}$. A detailed analysis of the island size and island density as a function of coverage by AFM and synchrotron experiments fully confirmed scaling predictions of the DLA theory [18] [19] and allowed to determine the stable nuclei size to four pentacene molecules [20]. If the growth temperature is lowered to $0^{\circ} \mathrm{C}$, the diffusion length is reduced by a factor $\sim 4$ [20].

Pentacene submonolayer islands exhibit a layer thickness of $15.4 \AA$ which has been measured by AFM and by x-ray reflectometry [17]. This layer thickness implies that the molecules are oriented in an upright configuration, i.e. the long molecular axis is oriented predominantly along the surface normal, see Fig. 2a. Multilayer films exhibit different crystalline phases, which are usually identified by their $d_{001}$ spacing along the surface normal. The substrate-induced $\mathrm{d}_{001}=15.4 \AA$ polymorph, which is commonly termed thin film phase, is the most relevant for OTFT applications. Grazing incidence in plane diffraction experiments [21] [22] confirmed that the grains of the thin thin film phase are (001) oriented. The lateral dimension of the two dimensional unit cell inferred from these experiments, as well as the observed selection rules, suggest a unit cell with two non-equivalent molecules in a Herringbone arrangement, similar to the bulk pentacene ordering motive [23] [24].

In a recent study, the detailed molecular arrangement of the thin film phase was resolved by a grazing incidence truncation rod scattering study [25]. The crystal structure was found to be triclinic [26] with the following unit cell parameters: $\mathrm{a}=5.958 \pm$ $0.005 \AA, \mathrm{b}=7.596 \pm 0.008 \AA, \mathrm{c}=15.61 \pm 0.01$ $\AA, \alpha=81.25 \pm 0.04^{\circ}, \beta=86.56 \pm 0.04^{\circ}$ and $\gamma=$ $89.80 \pm 0.10^{\circ}$ [27]. A detailed analysis of the Bragg peak intensities for pentacene on $\mathrm{SiO}_{2}$ allowed to determine the tilt between the long molecular axis of the two unit cell molecules and the surface normal to $5.6 \pm 0.05^{\circ}$ and $6.0 \pm 0.4^{\circ}$ and the Herringbone angle to $54.3 \pm 1.3^{\circ}$ [27]. The unit cell is shown in Fig. 2b). Here, the unit cell vectors a (red), b (green) and c (blue) are color coded. The Herringbone angle (green arc) is defined as the intersection angle between molecular planes (red planes). In one case, also a molecular axis (red dotted line) is indicated.

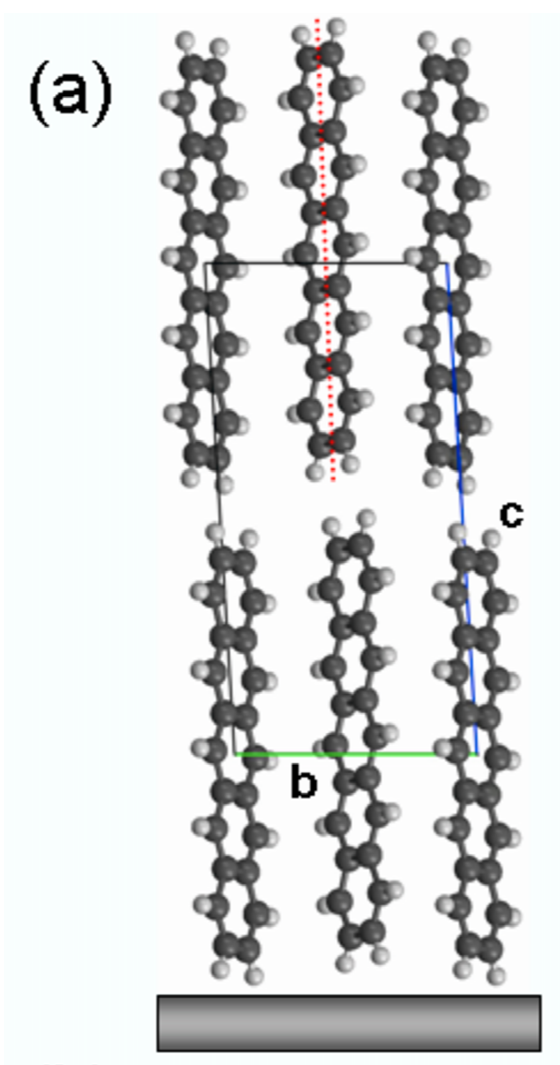

(b)

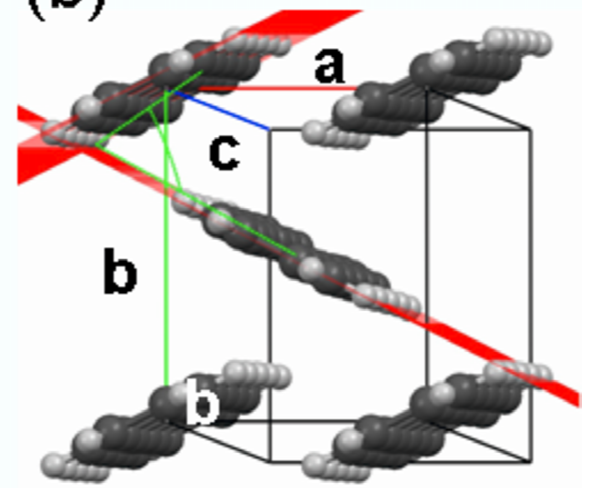

Fig. 2 Thin film phase unit cell. (a) The side view illustrates the layered structure of the thin film phase). (b) The top view emphasizes the herringbone ordering motive, which is a common feature of all pentacene polymorphs.

(c) 2003 WILEY-VCH Verlag GmbH \& Co. KGaA, Weinheim 
Surface energy calculations [28] reveal that the (001) cleaving plane is the surface with the lowest surface energy. In turn, the formation of (001) oriented films can be expected, if the interaction of the pentacene molecules with the surface is negligible to the pentacene-pentacene interaction. Experiments show that this condition apparently fulfilled for various inert substrates such as reduced and oxidized $\mathrm{Si}$, as well as many polymeric films used as gate dielectric. Note that the surface energy of the thin film phase is rather isotropic within the pentacene film plane [28], probably a direct result of the large herringbone angle. In turn, pentacene islands show on $\mathrm{SiO}_{2}$ a rather isotropic shape, thus favoring the formation of closed films up to several monolayers [16] [29]. Beyond this thickness, strong roughening occurs [30] and competing phases start nucleating [31].

2.2 Film formation on metallic and conductive surfaces

Bottom contact TFTs involve either metallic or conducting polymer contacts. Therefore, the growth of pentacene on metallic surfaces has also been studied in detail[[32],[33],[34],[35]]. The most commonly used contact material for pentacene TFTs is $\mathrm{Au}$, whose work function value $\phi=5.1 \mathrm{eV}$ [36] matches quite well with the ionization energy of pentacene $\chi=4.9 \mathrm{eV}$ [37]. Also, being a noble metal, Au contacts are sometimes considered insensitive to air exposure even if it has been observed that the $\mathrm{Au}$ work function is quite sensitive to ambient conditions. The observed growth behavior for pentacene on Au depends strongly on the substrate, i.e. different growth is observed for single Au crystal surfaces [32] and for thin Au film composed out of polycrystalline grains [33]. Also the roughness and cleaning procedure influence the growth. For the technically most relevant polycrystalline Au film surfaces, pentacene shows a complex growth behavior. If a $\mathrm{Au}(111)$ texture is present, a flat lying monolayer forms, which can be interpreted as a wetting layer. This layer then acts as a growth template for subsequently deposited pentacene forming microscopic grains. For these grains, the long molecular axis is oriented along the surface (lying down phase). Elevated temperatures promote a pronounced dewetting [34]. This behavior is not unexpected, since the surface energy of a lying down phase is higher than the surface energy of the thin film phase [28] and thus dewetting is a direct result of the minimization of the film surface area. Thus, surface diffusion is counterproductive for the formation of closed films and the formation of well ordered closed films seems quite impossible. To resolve this problem, two strategies have been pursued. One approach, which has been demonstrated for single crystal $\operatorname{Ag}(111)$ surfaces makes use of cryogenic substrate temperatures to suppress dewetting. In this case, the pentacene molecules are deposited making use of a hyperthermal He-beam [38] which provides the impinging pentacene molecules with energies up to $5 \mathrm{eV}$, well above thermal energies. Thus, a well ordered film can form at 200 K [35] (see Fig. 3a).

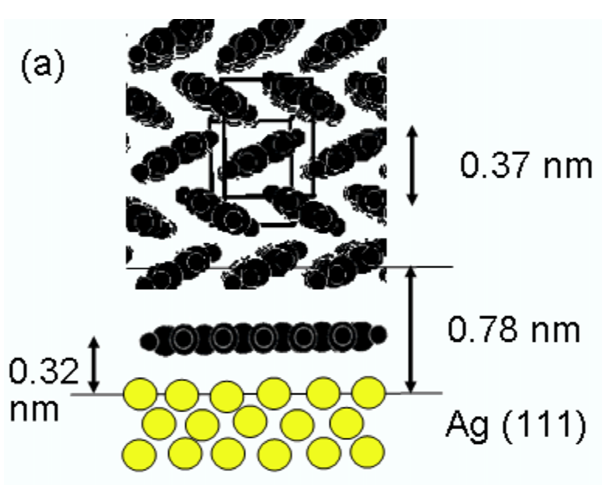

(b)

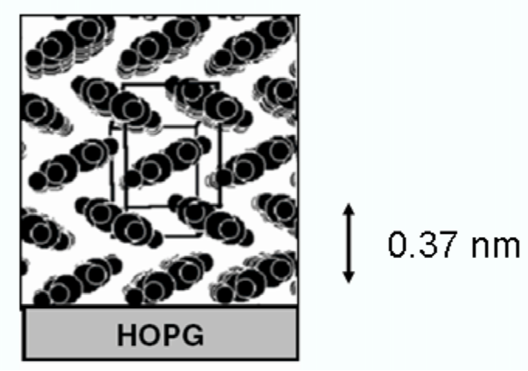

Fig. 3 Pentacene growth on Ag and graphite (a) Side view of the film structure of pentacene on $\operatorname{Ag}(111)$. A flat lying pentacene monolayer acts as a growth template. (b) Side view of the growth of pentacene on graphite. The structure resembles the growth on $\mathrm{Ag}$, but it is not clear whether a pentacene wetting layer is present.

Another way to avoid dewetting is to passivate the metallic surface by a self assembled monolayer (SAM), e.g. an alkane thiol monolayer (C-18). After passivation, the growth structure resembles the 
growth mode on inert surfaces [33], the same holds for growth of pentacene on conducting polymers such as PEDOT : PSS [poly(3,4-ethylenedioxythiophene) / poly(styrenesulfonate] [39]. It is interesting to note that on bare $\mathrm{Si}$, pentacene initially forms a flat lying monolayer, but on-top of this monolayer, the thin film phase readily forms [16] without need for passivation.

Other more exotic materials which have been studied include graphite and Bi. Graphite is an interesting substrate since it also promotes the formation of a lying down phase with Herringbone order textured in the (200) orientation [40] (see Fig. 3b). This enabled an angular resolved photoelectron emission study (APES) for this pentacene polymorph along the (200) direction of the Brillouin zone. The experiments reveal indications of strong band dispersion [40]. Recently, a well defined epitaxial relationship between a pentacene film and the $\mathrm{Bi}$ substrate has been realized. This well defined epitaxial relationship allowed to explore the band dispersion of different crystallographic directions of the Brillouin zone of pentacene by APES, also revealing a pronounced band dispersion [41].

\subsection{Mixed films}

For some applications such as organic photovoltaic devices or ambipolar transistors, a co-deposition of hole and electron conducting materials is needed, e.g. pentacene and C60. This gives rise to a whole zoo of possible growth scenarios, depending on how the different materials mix. For the co-deposition of pentacene and 6,13-pentacenequinone (an oxidized state of pentacene) a pronounced phase separation is observed, depending on deposition rates [42]. At the same time, the formation of the pentacene bulk phase is suppressed in mixed film.

\section{Pentacene OTFT properties}

\subsection{Mobility and charge carrier density}

The most simple pentacene OTFT test structure used in many labs is based on a Si wafer piece covered with a thermal oxide. Here, the heavily doped $\mathrm{Si}$ wafer takes the role of the back gate electrode, and the $\mathrm{SiO}_{2}$ takes the role of the gate dielectric. A pentacene thin film is deposited as the semiconducting layer. Source and drain electrodes are deposited either on the silicon oxide (bottom contact) or on top of the pentacene film (top contact).

A pentacene TFT works in hole accumulation, i.e. a sufficiently negative voltage $\left(\mathrm{V}_{G}\right)$ is applied to the gate, accumulating holes at the pentacene $/ \mathrm{SiO}_{2}$ interface. If now a moderate voltage is applied between the source and drain electrode, a hole current flows. A characteristic output curve for a typical pentacene TFT from our lab is shown in Fig. 4a. Here, as a substrate, heavily n-doped Si-wafers with a $150 \mathrm{~nm}$ thick thermal $\mathrm{SiO} 2$ layer on top of it were used. Additionally, a very thin layer of polysterene (ca. $2 \mathrm{~nm}-3 \mathrm{~nm}$ ) was spin cast on top of the silicon oxide layer. A shadow mask was used to define $50 \mathrm{~nm}$ thick source and drain Au contacts that were evaporated in vacuum. Finally a $50 \mathrm{~nm}$ pentacene layer was evaporated at room temperature at a deposition rate of about $1 \mathrm{~nm} /$ minute in a separate evaporation chamber, also using a shadow mask. This way, transistor channels with a length of $\mathrm{L}=25 \mu \mathrm{m}$ and a width of $\mathrm{W}=1000 \mu \mathrm{m}$ were obtained, $\mathrm{cf}$. schematic in Fig. 4. The output curve of this device shows a linear increase of the drain current for small drain voltages, and subsequently saturation occurs. The transfer characteristics of this device are shown in Fig. 4b. The logarithmic scale reveals a sharp onset of the transistor activity and a subthreshold swing $S=V_{G} / \log \left(I_{S} D\right)=1.1 \mathrm{~V}$ per decade.

Pentacene OTFT curves are usually analyzed within MOSFET theory [43]. For the geometry of the device used in Fig. 4, which has a channel width (W= $1000 \mu \mathrm{m}$ and a channel length $\mathrm{L}=25 \mu \mathrm{m}$, the sourcedrain current $\left(\mathrm{I}_{S D}\right)$ in the linear regime $\left(V_{S D}<<\mathrm{V}_{G}-\mathrm{V}_{T}\right)$ can be written as:

$$
I_{S D}^{l i n}=W / L \cdot \mu^{l i n} \cdot C_{i} \cdot\left(V_{G}-V_{T}\right) \cdot V_{S D}
$$

Here, the graded channel approximation has been used. $C_{i}$ is the capacitance per unit area of the dielectric and $\mathrm{V}_{T}$ is the threshold voltage. $\mu^{\text {lin }}$ is the mobility in the linear regime. The respective measurements are shown in Fig. 5a.

For the saturation regime $\left(V_{S D}>>\mathrm{V}_{G}-\mathrm{V}_{T}\right)$ the accumulation within the channel is incomplete. This so called pinch off arises due to the superposition of the gate and drain potential. The source-drain current $\left(\mathrm{I}_{S D}\right)$ in the saturation regime reads:

$$
I_{S D}^{S a t}=W /(2 L) \cdot \mu^{s a t} \cdot C_{i} \cdot\left(V_{G}-V_{T}\right)^{2}
$$




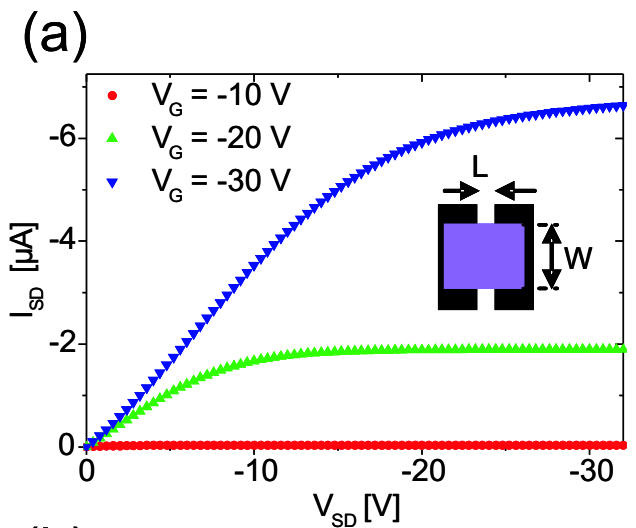

(b)

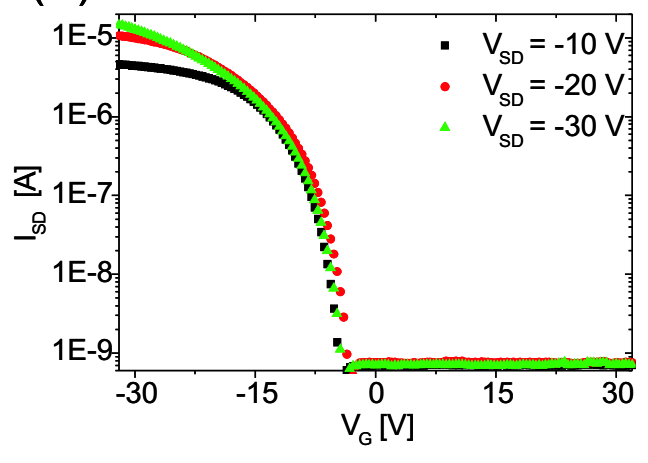

Fig. 4 Characteristics of a bottom contact pentacene OTFT. Channel geometry: $\mathrm{L}=$ $25 \mu \mathrm{m}, \mathrm{W}=1000 \mu \mathrm{m}$ (see text and inset). Sweep rate: $0.4 \mathrm{~V} / \mathrm{s}$, the measurement was performed in vacuum. (a) Output characteristics $\left[\mathrm{I}_{S D}\right.$ vs $\mathrm{V}_{S D}, \mathrm{~V}_{G}$ as indicated in the inset]. (b) and Transfer characteristics [ $\mathrm{I}_{S D}$ vs $\mathrm{V}_{G}$ in $\log$. scale, $\mathrm{V}_{S D}$ as indicated in the inset].

The respective measurements are shown in Fig. 5 b. The mobility in the saturated regime $\left(\mu^{\text {sat }}\right)$ can be read off from the slope of the output curve (straight lines in Fig. 5b, square root scale at y-axis) using Eq. (2). We obtain $\mu^{\text {sat }}=0.06 \mathrm{~cm}^{2} /(V s)$, which compares reasonably well to typical values for the bottom contact geometry $\left(\mu \approx 0.1-0.3 \mathrm{~cm}^{2} /(V s)\right.$ ). The mobility in the linear regime $\left(\mu^{\text {lin }}\right)$ can be read off from the slope of the output curve (Fig. 5a, linear scale at $y$-axis) using Eq. (1). We obtain $\mu^{\text {lin }}=$ $0.04 \mathrm{~cm}^{2} /(V s)$, which is slightly reduced compared to $\mu^{\text {sat }}=0.06 \mathrm{~cm}^{2} /(V s)$, indicating some nonideal behavior. (a)

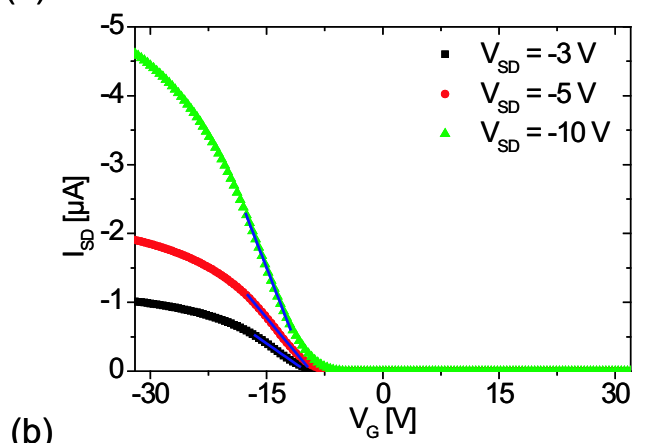

(b)

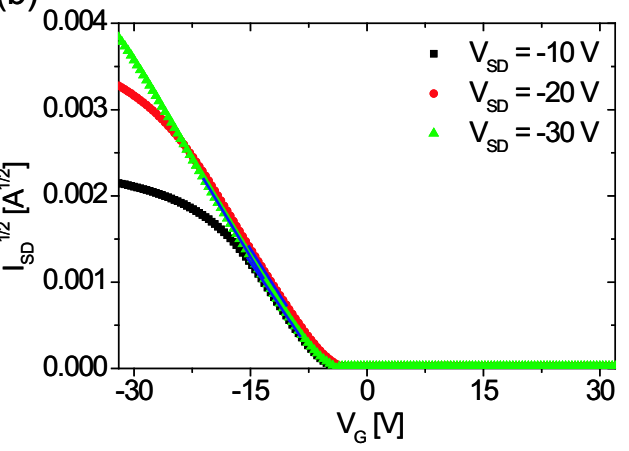

Fig. 5 Transfer characteristics. (a) Linear regime $\left(\mathrm{I}_{S D}\right.$ vs $\left.\mathrm{V}_{G}\right)$, the straight lines indicate the slope determining $\mu^{\text {lin }}$ according to Eq. (1). (b) Saturated regime $\left(\sqrt{I_{S D}}\right.$ vs $\left.\mathrm{V}_{G}\right)$, the straight lines indicate the slope determining $\mu^{\text {sat }}$ according to Eq. (2). Same device as in Fig. 4.

The most important physical parameter of an OTFT beyond the mobility $\mu$ is the number of charge carriers $\left(\mathrm{n}_{h}\right)$ induced by a given gate voltage $\left(\mathrm{V}_{G}\right) \cdot \mathrm{n}_{h}$ can be estimated from the measured channel conductivity $\sigma$ by

$$
n_{h} \cdot q \cdot \mu=\sigma / d=L /(W \cdot R)
$$

Here, $d$ is the active channel thickness (1-2 molecular layers) and $q$ is the elementary charge of a hole. For the transistor used in Fig. 4, we measure $R=2.8 \cdot 10^{6} \Omega$ at $\mathrm{V}_{G}=-30 \mathrm{~V}$ and $\mathrm{V}_{S D}=-10 \mathrm{~V}$. In turn, according to Eq. (3), the hole carrier density at this set point is $n_{h}=0.9 \cdot 10^{12} \mathrm{~cm}^{-2}$.

3.2 Influence of trap states and fixed interface charges

An example for the dynamic behavior of a pentacene OTFT is shown in Fig. 6a. Here, the gate voltage 
$\left(\mathrm{V}_{G}\right)$ is first swept from positive to negative voltages (sweep rate $=0.4 \mathrm{~V} / \mathrm{s}$, black data points in Fig. $6 \mathrm{a})$, and then immediately from negative to positive voltages (red data points in Fig. 6a) for the same fixed value of the drain voltage $\left(\mathrm{V}_{S D}=-20 \mathrm{~V}\right)$. A pronounced hysteresis is observed. This hysteresis can be interpreted as an apparent shift of the threshold voltage $\left(\Delta \mathrm{V}_{T}\right)$ induced by a charging $Q_{t}$ of the pentacene $/ \mathrm{SiO}_{2}$ interface due to filling and emptying of trap states. The number of trap states can be estimated by

$$
n_{t}=Q_{t} / q=\Delta V \cdot C_{i} / q
$$

The observed threshold shift of $\Delta \mathrm{V}_{T}=2.0 \mathrm{~V}$ results in a trap density $\mathrm{n}_{t}=2.4 \cdot 10^{11}$ traps $/ \mathrm{cm}^{2}$. This number is not so small compared to the number of charge carriers $n_{h}$, indicating that traps largely influence the device performance. One may speculate whether structural defects within the pentacene film contribute to trap states. An x-ray analysis of the defect densities in pentacene films revealed defect densities in the order of $\mathrm{n}_{t}=2 \cdot 10^{11}$ defects $/ \mathrm{cm}^{2}$ [44], in good agreement with the trap density $\mathrm{n}_{t}$ inferred from the electronic characterization. Thus, the observed structural defects apparently contribute to trap states. It is well known, that the hysteresis and thus the trap density depends on the nature of the gate dielectric surface. Especially hydroxyl groups are considered as potential trap states, therefore a passivation of the gate oxide by either silanization [45] or polymer capping (here: polystyrene film) is needed to minimize trap densities.

A subsequent study of the pentacene TFT reveals also an irreversible drift of the transfer curve with operating time, cf. Fig. 6b. This drift can be interpreted as an interface charging due to deep trap levels, also termed fixed charges. The observed voltage shift of $\Delta \mathrm{V}=6.5 \mathrm{~V}$ implies a fixed charge density $n_{d t}=7.7 \cdot 10^{11} \mathrm{~cm}^{-2}$. Note that this density is already of the order of the charge carrier density $\mathrm{n}_{h}$. In turn, voltage shifts in pentacene TFTs can completely redefine the working point of a transistor. For the application of such TFT devices in e.g. ring oscillators [46], one should find a way to control these drifts. Recent work suggests that controlled generation of fixed charges at the gate dielectric by UH-light exposure might be used to store electronically readable information in a pentacene TFT (one bit per transistor). (a)
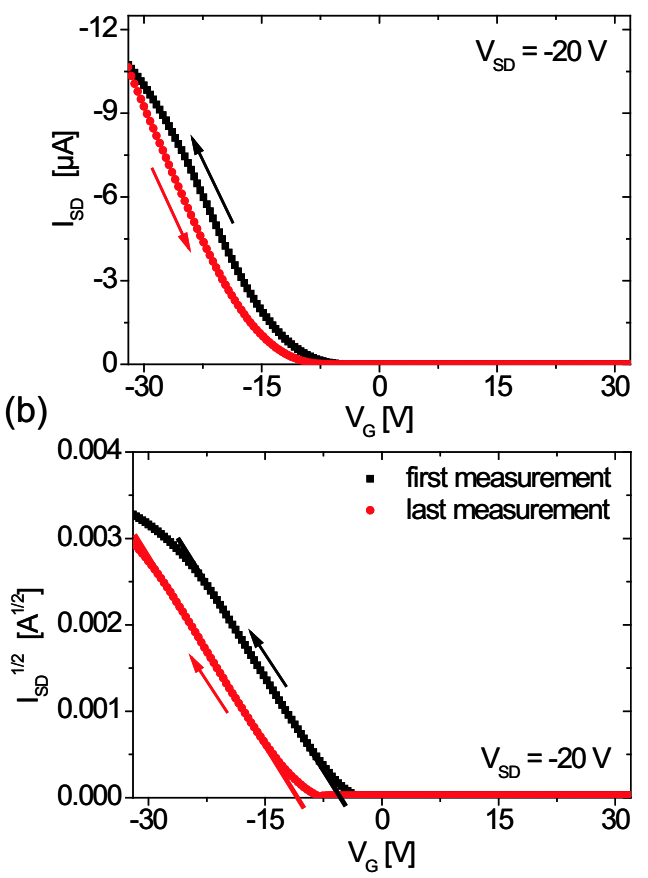

Fig. 6 Hysteresis effects and voltage drifts in the transfer characteristics. Sweep rate is 0.4 $\mathrm{V} / \mathrm{s}$, same device as in Fig. 4. (a) A gate sweep from positive to negative voltages (black data points), and back (red data points) for the same fixed value of the drain voltage $\left(\mathrm{V}_{S D}=-20\right.$ V) reveals a hysteresis effect. (b) After a couple of measurements (one hour later), a rather stable shift of the transfer curve due to fixed charges is established for the same device.

\subsection{Injection}

The whole TFT performance is largely influenced by injection efficiencies. In particular surface contaminations such as remanent photo resist or organic adsorbates due to air or solvent exposure can modify the work function of the Au electrode up to a point, that charge injection is locally suppressed. The injection properties of two selected TFT devices from our lab are compared in Fig. 7. Both devices are operated at $\mathrm{V}_{G}=-30 \mathrm{~V}$. The output characteristics of the two devices show a pronounced difference for small drain voltage $\mathrm{V}_{S D}$. While one device (so called good device, black data points in Fig. 7) shows an ohmic increase of $\mathrm{I}_{S D}$ as a function of $\mathrm{V}_{S D}$, the other device shows a damped response (so 
called bad device, red data points in Fig. 7). Note that the amplitude of $\mathrm{I}_{S D}$ of the bad device has been rescaled for better comparison. Both devices are bottom contact TFTs of similar geometry, however, the preparation of the contacts was different. The contacts of the good device were fabricated using a shadow mask, while the contacts of the bad device were made produced using optical lithography. Optical lithography involves resist and use of solvents such as acetone, which apparently can lead to less efficient contact properties. The physical mechanism which gives rise to the non-ohmic behavior remains unclear at this level of analysis and thus using equivalent circuits [47] which take account of the observed deviations from ideal MOSFET behavior seem to be the most practical way to deal with these effects.

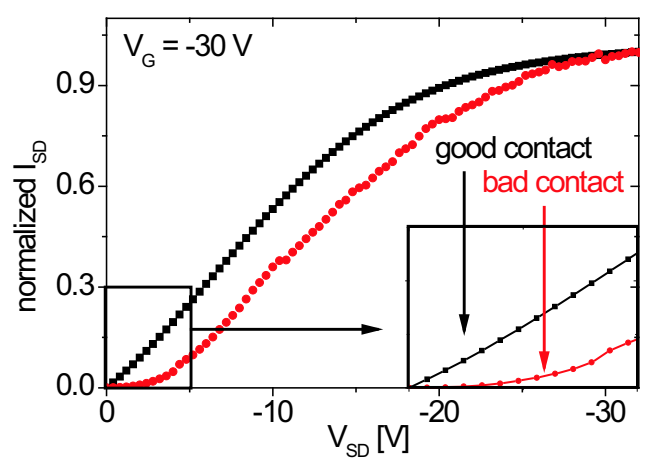

Fig. 7 Injection efficiencies of pentacene TFTs. Comparison of ohmic and non-ohmic behavior. The contacts of the so called good device were fabricated using shadow masks, and the contacts of the so called bad device were fabricated using optical lithography.

\section{Photoresponse}

Pentacene shows a strong absorption in the visible (see Fig. 8), more optical properties of pentacene films can be found in [48]. If pentacene is combined with a proper n-conductor such as $\mathrm{C} 60$, it can be used as active region in a solar cell [49]. Transient photoconductivity experiments using optical pump terahertz probe techniques suggest that charge photo generation occurs in pentacene on a subpicosecond timescale [50].

Here, we employ photo generated electron-hole pairs to analyze local properties of a TFT device.

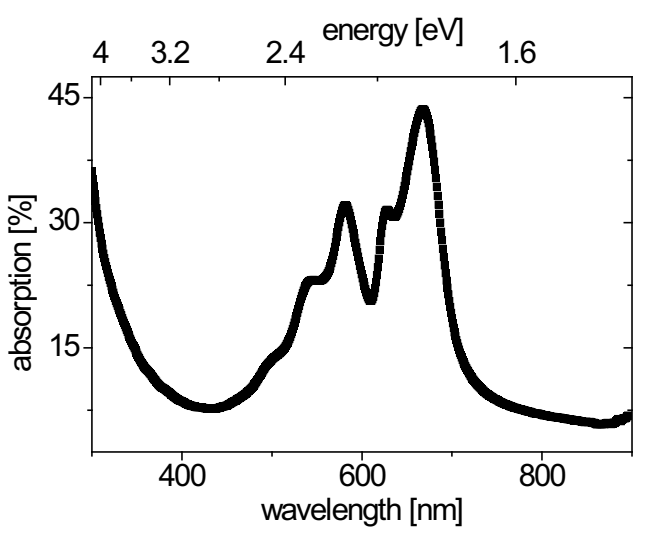

Fig. 8 Absorption spectra. Wavelength dependency of the absorption of a $50 \mathrm{~nm}$ pentacene thin film on glass.

For this purpose, we have adapted a laser scanning confocal microscope experiment to spatially resolve the photo response of a TFT. The experimental setup is shown schematically in Fig. 9a. The laser spot size on the TFT is less than $1 \mu \mathrm{m}$. A $\mathrm{x}-\mathrm{y}-\mathrm{z}$ piezopositioner stage [51] which is mounted on top of a $\mathrm{x}-\mathrm{y}$ translation stage is used to translate the sample with respect to the laser. The reflected beam allows to identify the contact and the channel region. The laser beam is modulated by a chopper, and the difference signal is extracted using a lock-in amplifier.

We observe a strong photo response localized at the anode [52]. The response is inhomogenous along the contact indicating variations in the transport or injection properties of the device. These variations may be due to local variations of the contact work function, or due to bad physical contact of the pentacene grains adjacent to the electrode, or due to a local enhancement of defect densities in the pentacene film. Thus, a systematic study of the photo response in combination with the respective characteristic transistor curves allows visualizing problematic regions of an OTFT, which is a key prerequisite for device optimization.

\section{Outlook}

Pentacene has all the qualities needed for an OTFT. The main challenge is the control of the contact and dielectric interfaces to suppress the voltage drifts and hysteresis effects associated with trap states. Also 


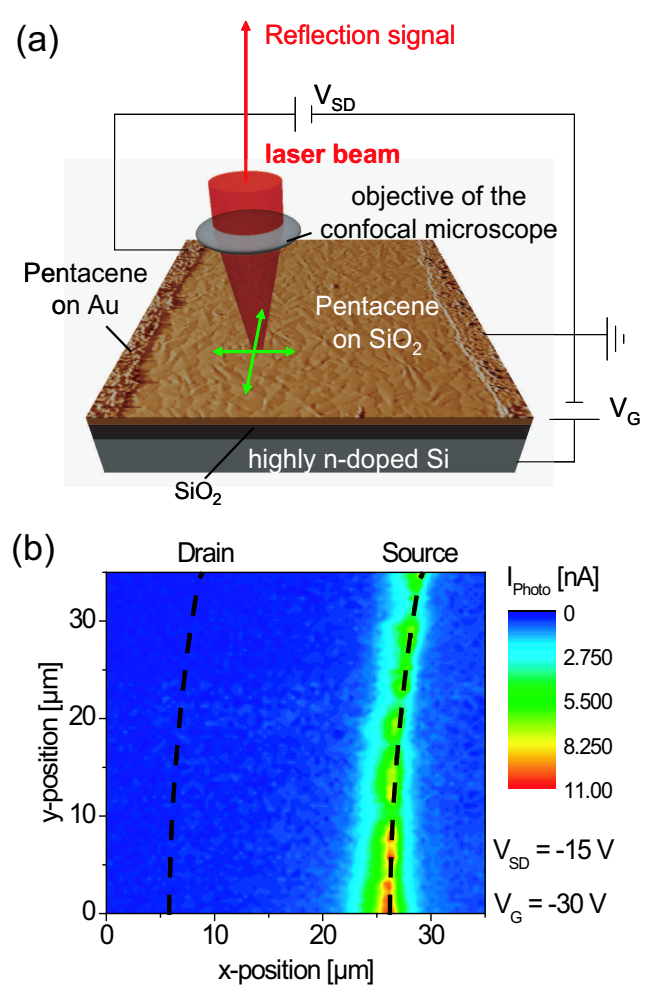

Fig. 9 Spatially resolved photo response experiment. (a) Schematic of the experimental setup for spatially resolved photo response measurements. (b) Spatially resolved photo response data in the linear regime $\left(\mathrm{V}_{G}=-30 \mathrm{~V}\right.$ and $\left.\mathrm{V}_{S D}=-15 \mathrm{~V}\right)$. A Corbino device structure has been used (disk and ring geometry, $\mathrm{L}=$ $20 \mu \mathrm{m}$ [channel length], $\mathrm{W}=1005 \mu \mathrm{m}$ [channel width, i.e. circumference]). The photo response measurement covers a small fraction of the whole device.

the dependency of the injection efficiencies on contact preparation suggest that even if pentacene deposition technology can be applied under rather rough conditions, ultra high vacuum and clean environments may still be useful on the way of learning how to produce reliable devices.

Acknowledgements $\mathrm{BN}$ acknowledges financial support by Deutsche Forschungsgemeinschaft under contract no. Ni 632/1-1 (research fellowship at Princeton U.) and Ni632/2-1 (research proposal within SPP1121) and hospitality from G. Scoles and J. Rädler. We also acknowledge financial support by Elite Netzwerk Bayern, CeNS and NIM. Purified pentacene was received from J. Pflaum, Universität Stuttgart. The x-ray experiments were performed at NSLS in Brookhaven and at HASYLAB in Hamburg. We are grateful to J. Kotthaus for providing access to clean room facilities at LMU. Finally, it is a pleasure to acknowledge fruitful discussions with the participants of SPP1112, and with N. Koch and U. Beierlein.

\section{References}

[1] G.G. Malliaras and R. Friend, An Organic Electronics Primer, Physics Today May, (2005).

[2] S. R. Forrest, The path to ubiquitous and low-cost organic electronic appliances on plastic, Nature 428,(6986) 911-918 (2004).

[3] C. D. Dimitrakopoulos, A. R. Brown, and A. Pomp, Molecular beam deposited thin films of pentacene for organic field effect transistor applications, Journal Of Applied Physics 80,(4) 2501-2508 (1996).

[4] D.J. Gundlach, Y.Y. Lin, T.N. Jackson, S.F. Nelson, and D.G. Schlom, Pentacene organic thin-film transistors-molecular ordering and mobility, IEEE Electron Device Letters 18,(3) 87-89 (1997).

[5] Y. Y. Lin, D. J. Gundlach, S. F. Nelson, and T. N. Jackson, Stacked pentacene layer organic thin-film transistors with improved characteristics, Ieee Electron Device Letters 18,(12) 606-608 (1997).

[6] D. Knipp, R. A. Street, A. Volkel, and J. Ho, Pentacene thin film transistors on inorganic dielectrics: Morphology, structural properties, and electronic transport, Journal of Applied Physics 93,(1) 347355 (2003).

[7] C. D. Dimitrakopoulos and D. J. Mascaro, Organic thin-film transistors: A review of recent advances, Ibm Journal Of Research And Development 45,(1) 11-27 (2001).

[8] C. D. Dimitrakopoulos and P. R. L. Malenfant, Organic Thin Film Transistors for Large Area Electronics, Advanced Materials 14,(2) 99-117 (2002).

[9] G. R. Desiraju and A. Gavezzotti, CrystalStructures Of Polynuclear Aromatic-Hydrocarbons Classification, Rationalization And Prediction From Molecular-Structure, Acta Crystallographica Section B-Structural Science 45, 473-482 (1989).

[10] R. Ruiz, D. Choudhary, B. Nickel, T. Toccoli, K.-C. Chang, A.C. Mayer, P. Clancy, J.M. Blakely, R.L. Headrick, S. Iannotta, Malliaras G.G., B. Nickel, T. Toccoli, K.-C. Chang, A.C. Mayer, P. Clancy, J.M. Blakely, R.L. Headrick, S. Iannotta, and G.G. Malliaras, Pentacene thin film growth, Chemistry of Materials Special Issue on Organic Electronics, (2004).

[11] G. Horowitz and P. Delannoy, An Analytical Model for Organic-Based Thin-Film Transistors, Journal of Applied Physics 70,(1) 469 (1991). 
[12] G. Gu, M. G. Kane, J. E. Doty, and A. H. Firester, Electron traps and hysteresis in pentacene-based organic thin-film transistors, Applied Physics Letters 87,(24) (2005).

[13] S. R. Forrest, Ultrathin organic films grown by organic molecular beam deposition and related techniques, Chemical Reviews 97,(6) 1793-1896 (1997).

[14] G. B. Blanchet, C. R. Fincher, and I. Malajovich, Laser evaporation and the production of pentacene films, Journal of Applied Physics 94,(9) 6181-6184 (2003).

[15] J. A. Venables, G. D. T. Spiller, and M. Hanbrcken, Nucleation and growth of thin films, Rep. Prog. Phys. 47, 339-459 (1984).

[16] Frank-J. Meyer Zu Heringdorf, M. C. Reuter, and R. M. Tromp, Growth dynamics of pentacene thin films, Nature 412, 517-520 (2001).

[17] R. Ruiz, B. Nickel, N. Koch, L. C. Feldman, R. F. Haglund, A. Kahn, and G. Scoles, Pentacene ultrathin film formation on reduced and oxidized Si surfaces, Physical Review B 67,(12) 125406 (2003).

[18] J. G. Amar, F. Family, and Pui-Man Lam, Dynamic scaling of the island-size distribution and percolation in a model of submonolayer molecular-beam epitaxy, Physical Review B 50,(12) 8781-8797 (1994).

[19] J. G. Amar and F. Family, Critical Cluster Size: Island Morphology and Size Distribution in Submonolayer Epitaxial Growth, Physical Review Letters 74,(11) 2066-2069 (1995)

[20] R. Ruiz, B. Nickel, N. Koch, L. C. Feldman, R. F. Haglund, A. Kahn, and G. Scoles, Dynamic Scaling, Island size distribution and Morphology in the Aggregation Regime of Sub-monolayer Pentacene films, Physical Review Letters 91, 136102 (2003).

[21] S. E. Fritz, S. M. Martin, C. D. Frisbie, M. D. Ward, and M. F. Toney, Structural characterization of a pentacene monolayer on an amorphous SiO2 substrate with grazing incidence $X$-ray diffraction, Journal of the American Chemical Society 126,(13) 4084 (2004).

[22] A. C. Malliaras G. G. Nickel B. Scoles G. Kazimirov A. Kim H. Headrick R. L. Islam Z. Ruiz, R. Mayer, Structure of pentacene thin films, Applied Physics Letters 85,(21) 4926 (2004).

[23] R. B. Campbell, J. Trotter, and J. M. Robertson, Crystal and molecular structure of pentacene, Acta crystallographica 14,(7) 705 (1961).

[24] R. B. Campbell, J. Trotter, and J. Moneath, Crystal structure of hexane, and a revision of crystalographic data for tertacene and pentacene, Acta crystallographica 15,(3) 289 (1962).

[25] R. Feidenhansl, Surface structure determination by $x$-ray diffraction, Surface Science Reports 10,(3) 105-188 (1989).
[26] H. Yoshida and N. Inaba, K.and Sato, X-ray diffraction reciprocal space mapping study of the thin film phase of pentacene, Applied Physics Letters 90,(189) 181930 (2007).

[27] S. Schiefer, M. Huth, A. Dobrinevski, and B. Nickel, Determination of the crystal structure of substrateinduced pentacene polymorphs in fibre structured thin films, Journal of the American Chemical Society in press, (2007).

[28] J. E. Northrup, M. L. Tiago, and S. G Louie, Surface energetics and growth of pentacene, Physical Review B 66,(12) 121404 (2002).

[29] R. Ruiz, B. Nickel, N. Koch, L.C. Feldman, R.F. Haglund, A. Kahn, and G. Scoles, Pentacene ultrathin film formation on reduced and oxidized Si surfaces, Physical Review B (Condensed Matter and Materials Physics) 67,(12) 125406-1-7 (2003).

[30] S. Kowarik, A. Gerlach, W. Leitenberger, W. Hu, G. Witte, C. Wll, U. Pietsch, and F. Schreiber, Energy-dispersive $X$-ray reflectivity and GID for real-time growth studies of pentacene thin films, Thin Solid Films online available, (2007).

[31] A.C. Mayer, A. Kazimirov, and G.G. Malliaras, Dynamics of Bimodal Growth in Pentacene Thin Films, Physical Review Letters 97, 105503 (2006).

[32] D. Käfer and G. Witte, Evolution of pentacene films on $\mathrm{Ag}$ (111): Growth beyond the first monolayer, Chem. Phys. Lett. 442, 376 (2007).

[33] D. Käfer, L. Ruppel, and G. Witte, Growth of pentacene on clean and modified gold surfaces, Physical Review B 75, 085309 (2007).

[34] G. Beernink, T. Strunskus, G. Witte, and Ch. Wöll, Growth of conjugated oligomer thin films studied by atomic-force microscopy volume 85, (2004).

[35] L. Casalis, M. F. Danisman, B. Nickel, G. Bracco, T. Toccoli, S. Iannotta, and G. Scoles, Hyperthermal molecular beam deposition of highly ordered organic thin films, Physical Review Letters 90,(20) 206101 (2003).

[36] Handbook of chemistry and physics, CRC Press Cleveland, Ohio (1981).

[37] E. A. Silinsh and V. Ćápek, Organic molecular crystals: Interaction, Localization and Transport phenomena, AIP New York (1994).

[38] F. De Angelis, T. Toccoli, A. Pallaoro, N. Coppede, L. Mariucci, G. Fortunato, and S. Iannotta, SuMBE based organic thin film transistors, Synthetic Metals 146,(3) 291-295 (2004).

[39] N. Koch, B. Nickel, J. Ghijsen, A. Elschner, J. Schwartz, J.-J. Pireaux, and A. Kahn, Metal vs. Polymer Electrodes in Organic Devices: Energy Level Alignment, Hole Injection, and Structure, Mat. Res. Soc. Symp. Proc. 771,(20) (2003).

[40] N. Koch, A. Vollmer, I. Salzmann, B. Nickel, $\mathrm{H}$. Weiss, and J. P. Rabe, Evidence for temperaturedependent electron band dispersion in pentacene, Physical Review Letters 95,(15) 156803 (2006). 
[41] H. Kakuta, T. Hirahara, I. Matsuda, T. Nagao, S. Hasegawa, N. Ueno, and K. Sakamoto, Electronic Structures of the Highest Occupied Molecular Orbital Bands of a Pentacene Ultrathin Film, Phys. Review Lett. 98, 247601 (2007).

[42] I. Salzmann, S. Opitz, R.and Rogaschewski, J. P. Rabe, B. Nickel, and N. Koch, Phase separation in vacuum co-deposited pentacene/6,13pentacenequinone thin films, Physical Review B 75, 174108 (2007).

[43] S. M. Sze, Physics of semiconductor devices, Wiley Singapore (1981).

[44] B. Nickel, R. Barabash, R. Ruiz, N. Koch, A. Kahn, L. C. Feldman, R. F. Haglund, and G. Scoles, Dislocation arrangements in pentacene thin films, Physical Review B 70,(12) 125401 (2004).

[45] M. Shtein, J. Mapel, J. B. Benziger, and S. R. Forrest, Effects of film morphology and gate dielectric surface preparation on the electrical characteristics of organic-vapor-phase-deposited pentacene thin-film transistors, Applied Physics Letters 81,(2) 268 (2002).

[46] C. Erlen, P. Lugli, M. Fiebig, and B. Nickel, Transient TCAD simulation of three-stage organic ring oscillator, Journal of Computational Electronics 5,(4) 345 (2006).

[47] P. V. Necliudov, M. S. Shur, D. J. Gundlach, and T. N. Jackson, Modeling of organic thin film transistors of different designs, Journal Of Applied Physics 88,(11) 6594-6597 (2000).

[48] D. Faltermeier, B. Gompf, M. Dressel, A. K. Tripathi, and J. Pflaum, Optical properties of pentacene thin films and single crystals, Physical Review B 74,(12) 125416 (2006)

[49] S. Yoo, B. Domercq, and B. Kippelen, Efficient thinfilm organic solar cells based on pentacene/C60 heterojunctions, Applied physics letters 85,(22) 5427 (2004).

[50] O. Ostroverkhova, D. G. Cooke, F. A. Hegmann, J. E. Anthony, V. Podzorov, M. E. Gershenson, O. D. Jurchescu, and T. T. M. Palstra, Ultrafast carrier dynamics in pentacene, functionalized pentacene, tetracene, and rubrene single crystals, Applied physics letters 88,(12) 162101 (2006).

[51] Atto Cube Systems AG in München.

[52] M. Fiebig et al., submitted , (2007). 